\title{
Network performance evaluation algorithm based on BP neural network
}

\author{
Qi Liü, a , Xiyue Wang ${ }^{1, b}$, Yiyong Lin ${ }^{2, c}$, Ling He ${ }^{1, d}$, Yunzhi Huang ${ }^{1, e}$ \\ ${ }^{1}$ School of Electrical Engineering and Information, Sichuan University, Chengdu, China \\ ${ }^{2}$ Xichang Satellite Launch Center, Xichang, China \\ aliuqi@scu.edu.cn, ${ }^{b} 1067823139 @ q q . c o m,{ }^{c} 799729599 @ q q . c o m,{ }^{d}$ ling.he@scu.edu.cn, ehuang_y \\ unzhi@scu.edu.cn
}

\begin{abstract}
Keywords: Net performance evaluation BP neural network Cross validation
\end{abstract}
\begin{abstract}
In this paper, a network performance evaluation algorithm is proposed based on BP neural network. This system collects 8 network parameters in a local area network, and classifies the performance of network into three states: excellent, good and unqualified. Verification and analysis results show that the accuracy of the proposed network performance evaluation algorithm reaches up to $90 \%$ by $\mathrm{K}$-fold cross validation test. The experiments indicate that this proposed algorithm is robust and effective. Moreover, the calculation time of this system is less than $1 \mathrm{~ms}$. It could be effectively applied for a real-time network performance evaluation.
\end{abstract}

\section{Introduction}

The rapid development of network techniques and business brings heavy burden to the network, and makes a big challenge to the network performance. Therefore, the network performance analysis techniques emerge, which measure the quality of the network operation by measuring, analyzing and modeling the key indicator obtained from this network. The typical network performance indicators include time delay, network bandwidth, packet loss and so on. An effective network performance evaluation algorithm contributes to improve the overall performance of the network, and provides a more reasonable allocation of network resources.

Currently, many researchers have studied deeply on the methods of network performance evaluation. Yun Zhong et al. [1] have proposed a new method based on grey fuzzy to achieve network performance evaluation. After selecting key indicators of network performance, they used grey correlation analysis to obtain the correlation degree of each evaluation index. Then, the fuzzy relation matrix is constructed. After performing the fussy calculation, Yun Zhong et al. finally completes the performance evaluation and the ranking of the network object. Yang Nan et al. [2] put forward a network performance evaluation method. Yang Nan et al. determine the objective weights and compute performance weights dynamically. The data normalization method is utilized based on historical parameters, and the comprehensive network performance evaluation model is built. Huaqiong Zhao et al. [3] present a service-oriented network performance comprehensive evaluation model. The model takes application services as the guidance according to user preference. Firstly, an evaluation hierarchy is established, and the criteria weights and project weights in the hierarchy are determined respectively. Then the actual measurement data is normalized. Finally, the performance evaluation value of the target link is computed using fuzzy Analytic Hierarchy Process (AHP). Decebal Constantin Mocanu et al. [4,5] introduce a new network performance methodology based on the quality of experience benchmarks, whereby the quality of the service is estimated as it is perceived by the user. Xiaobo Yan et al. [6,7] propose an application oriented network performance evaluation model. This model combines the qualitative and quantitative evaluation method, giving four levels of network performance, and proposes a new network performance evaluation indicator, which is a cumulative network performance evaluation value.

BP Neural Network (NN) is an important data mining tool used for classification. It usually learns by samples. If $\mathrm{NN}$ is supplied with enough samples, it could perform classification. This paper presents a net performance evaluation algorithm based on BP neural network. The proposed system is 
implemented on the Microsoft Visual Studio 2012 platform. The system collects eight real-time network parameters: CPU utilization of switch, utilization of memory, network link-off, delay, delay jitter, bandwidth, bit rate of transmission, and bit rate of reception. Combined with BP neural network, three net performance levels are assessed: excellent, good or unqualified.

\section{Parameters of Local Area Network}

For different Local Area Network (LAN), the index parameters, which reflect the network performance, might be different. For example, for a remote medical communication network, the network delay and bandwidth are significant indicators in network performance evaluation. While for some public service networks, the network security and throughput are more important. The following principles should be obeyed while selecting the reasonable network performance parameters: simplicity, testability, and representation.

In the local area network, the CUP utilization of switch and memory utilization are important parameter for evaluating this type of network equipment. In network links, network link-off, delay, delay jitter, bandwidth, bit rate of transmission and bit rate of reception are important parameters. With full consideration of the local area network quality, this paper chooses the above eight parameters as the indicator parameters of system evaluation.

\section{BP neural network}

\section{Theory of BP neural network}

BP neural network is a kind of multilayer feedforward neural network and one of the most widely-used neural network models. Its learning strategy is fast descent method. And it adjusts the network weights and threshold by the back propagation to get the minimum error squares sum in the network. Topology of BP neural network includes input layer, hidden layer and output layer. Network learning is accomplished during the process of modifying the weights [8]. In this paper, the linear and S type excitation function are used [9]. The BP neural network topology is shown in Fig 1.

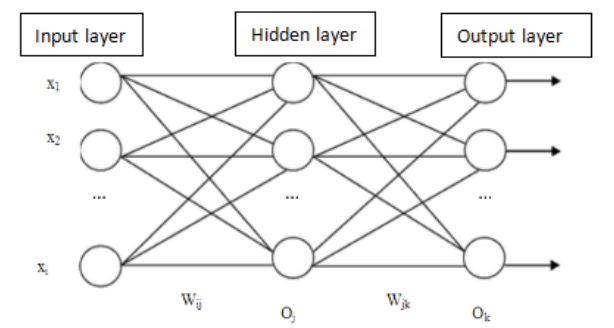

Fig 1 BP neural network topology

\section{Specific rules and relations}

Assume: The node number of input, hidden and output layer respectively are M, L and N. The node output of the input, hidden and output layer respectively are $\mathrm{O}_{\mathrm{i}}, \mathrm{O}_{\mathrm{j}}$ and $\mathrm{O}_{\mathrm{k}}$. The node input of the input, hidden and output layer respectively are net ${ }_{i}$ net $_{j}$ and net $t_{k}$. The node threshold of the input, hidden and output layer are $\theta_{i}, \theta_{j}$ and $\theta_{k}$ respectively. The weights of the hidden and output layer respectively are $\mathrm{w}_{\mathrm{ij}}$ and $\mathrm{w}_{\mathrm{jk}}$. The input of the neural network is $\mathrm{x}_{\mathrm{i}}$.

(1) Input and output of the $i^{\text {th }}$ node of the input layer:

$$
\begin{aligned}
& \text { net }_{i}=\sum_{i=1}^{M} x_{i}+\theta_{i} \quad i=1,2,3, \ldots, M \\
& o_{i}=f\left(\text { net }_{i}\right)=\frac{1}{1+\exp \left(- \text { net }_{i}\right)}
\end{aligned}
$$

(2) 
(2) Input and output of the $i^{\text {th }}$ node of the hidden layer:

$$
\begin{aligned}
n e t_{j} & =\sum_{j=1}^{N} w_{i j} o_{i}+\theta_{j} \\
o_{j} & =f\left(\text { net }_{j}\right)=\frac{1}{1+\exp \left(-n e t_{j}\right)}
\end{aligned}
$$

(3) Input and output of the $\mathrm{k}^{\text {th }}$ node in the output layer:

$$
\begin{aligned}
& n e t_{k}=\sum_{j=1}^{L} w_{j k} o_{j}+\theta_{k} \\
& o_{k}=f\left(n e t_{k}\right)=\frac{1}{1+\exp \left(-n e t_{k}\right)}
\end{aligned}
$$

(4) Weights adjustment

The weights of the output and hidden layer are corrected according to error gradient descent method to make the error cost be minimum. After joining momentum term [10], the weights' correction formula of the hidden layer and output layer are as follows:

$$
\begin{aligned}
& w_{i j}(k+1)=w_{i j}(k)+\eta_{j} \delta_{j} o_{i}+\alpha_{j}\left(w_{i j}(k)-w_{i j}(k-1)\right) \\
& w_{j k}(k+1)=w_{j k}(k)+\eta_{k} \delta_{k} o_{j}+\alpha_{k}\left(w_{j k}(k)-w_{j k}(k-1)\right)
\end{aligned}
$$

In the formula, $\eta$ and $\alpha$ are the coefficient of learning efficiency.

\section{Learning steps of BP neural network}

Step 1: Sample preparation. The eight parameters of network equipment and data link in the local area network are input samples. The output sample is automatically assessed into three levels: excellent, good and unqualified. The BP network is a network with six inputs and three outputs.

Step 2: Determine the initial parameters of the network. Including: Initial network weights and thresholds, determine the max training time, assign error cost function and set the cycle times.

Step 3: Compute the state of the output and hidden layer units.

Step 4: Calculate the error value of the output and hidden layer.

Step 5: Modify weights of the hidden layer and output layer.

Step 6: Judge whether the error cost function meeting the requirements. If reached, stop. Otherwise determine whether to achieve the number of cycle times, if achieved, stop, or turn to the second step.

The specific structure of the above algorithm is described in detail, which is showed in Fig 2:

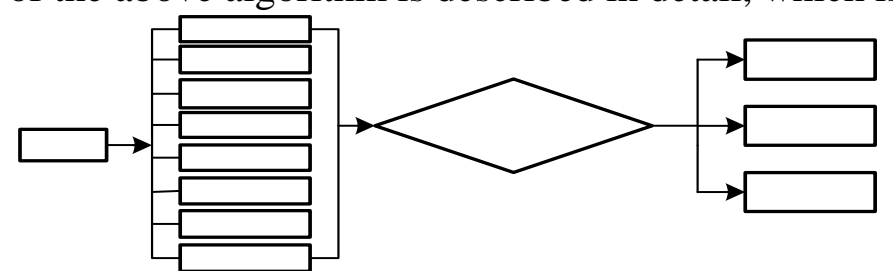

Fig 2 BP neural network algorithm

\section{Experiments and results}

\section{Training and testing datasets}

The testing local area network contains 89 switches and 168 data links. The proposed system is based on the Microsoft Studio Visual 2012 platform, using C++ programming language. The SNMP 
protocol in TCP/IP network protocol suite is implemented in this system to obtain the network parameters. The frequency of real-time data collection is $10 \mathrm{~s}$.

The presented system achieves real-time acquisition of network parameters, including two indicator parameters of switches and six indicator parameters of data links. The switches parameters are CPU utilization of switchboard and memory utilization. The link parameters are network link-off, delay, delay jitter, bandwidth, bit rate of transmission and bit rate of reception.

Under the excellent, good and unqualified network performance states, the eight parameters of network are collected 1000 times respectively. Thus, the database contains 3000 groups of network parameters, with equal number for three states of network performance (excellent, good and unqualified).

\section{Experiments and results}

The BP network system consists of six inputs, ten hidden neurons and three outputs. For the database illustrated in the above section, the proposed network performance evaluation method is applied, using k-fold cross validation algorithm. The correct assessment rate is $92 \%$.

This proposed system is tested on real-time application. The testing computer is with i7-6700k CPU, 4G graphics card and 8G memory. The BP neutral network model is built up using the collected database, which is illustrated in the above section. Then, the system collects the network parameters of all the switches and data links in the local area network, and the network performance is calculated using the proposed method. The consuming time is $0.5 \mathrm{~ms}$ for one time evaluation.

\section{Discussions and Conclusions}

This paper proposes a network performance evaluation algorithm based on the BP neural network. The accuracy of this presented method is over $90 \%$. It has short calculation time and can effectively realize real-time network performance evaluation of a local area network.

\section{References}

[1] Y. ZHONG, J.B. XIA and J.X WU: Journal of Air Force Engineering University: natural science edition, Vol.15 (2014) No 3, p.76.

[2] Y. NAN and L. CHEN: Journal of Computer Application, Vol.35 (2015) No 11, p.3055.

[3] H.Q. ZHAO and X.W. TANG: Journal of Computer Application, Vol.33 (2013) No 11, p.3015.

[4] D C MOCANU, G SANTANDREA, W CERRONI, F Callegati and A LIOTTA: 10th International Conference on Network and Service Management (CNSM) and Workshop (Rio de Janeiro, Brazil, November 17-21, 2014).

[5] A Liotta: Spectrum, IEEE, vol. 50 (2013) No 8, p. 26.

[6] X.B YAN, F. WANG and L. HU: International Journal of Online Engineering, vol. 9 (2103), pp. 51-56, 2013.

[7] A Manzalini, R Minerva, F CaUegati, W Cerroni, and A Campi: IEEE Communications Magazine, vol. 51 (2013) No 7, p. 63.

[8] L. LI, H.P. WU and Y. CHEN: China Water Transport, Vol.8 (2008) No 1, p.161.

[9] J.W. ZHUO: Application of MATLAB in Mathematical Modeling (Beihang University Press, China 2014).

[10]Y.B. HOU, J.Y. DU and M. WANG: Neural network (Xidian University Press, China, 2007). 